\title{
Analytical bit error rate performance of DS-CDMA ad hoc networks using large area synchronous spreading sequences
}

\author{
X. Liu, H. Wei and L. Hanzo
}

\begin{abstract}
The performance of large area synchronous (LAS) direct sequence code division multiple access (DS-CDMA) assisted ad hoc networks is investigated in the context of a single-hop infinite mesh of rectilinearly located ad hoc nodes. It is shown that LAS DS-CDMA exhibits a significantly better performance than the family of traditional spreading sequences used in a quasisynchronous DS-CDMA scenario having a low number of resolvable multipath components and a sufficiently high number of RAKE receiver branches. The benefits of LAS codes in ad hoc networks are multifold: (i) Their performance is noise-limited, rather than interference-limited, provided that the multipath and multi-user interference arrives within their interference free window. (ii) Under the same conditions LAS codes are robust against the 'near-far' effects imposed by ad hoc networks operating without base-station-aided power control, without accurate synchronisation and without implementationally complex interference cancellers.
\end{abstract}

\section{Introduction}

The family of large area synchronous (LAS) [1] spreading codes exhibits an interference free window (IFW), where the off-peak aperiodic auto-correlations and crosscorrelations become zero [2]. These attractive correlation properties assist us in eliminating the effects of both the multipath interference (MPI) and multiple access interference (MAI). Initial comparative studies between LAS-CDMA and traditional code division multiple access (CDMA) were presented, for example in [1].

LAS codes are generated by combining large area (LA) $[3,4]$ codes and loosely synchronous (LS) $[4,5]$ codes. $\mathrm{Li}$ [3] proposed various construction schemes for LA codes and analysed the performance of LA-CDMA, which exhibited higher spectral efficiency than traditional CDMA. Stanczak et al. [5] focused their attention on contriving systematic methods for the construction of LS codes. Choi and Hanzo [6] investigated the design of efficient and flexible LAS codes having an increased duty ratio, which resulted in a higher number of codes and hence a higher number of supported users than previous designs. An entire LAS-CDMA network was studied in [7]. For the detailed construction methods of LA, LS and LAS codes please refer to [3-5].

Again, as a benefit of having an IFW, LAS codes have the ability to support asynchronous operation in ad hoc networks, provided that both the propagation delay and the multipath-induced dispersion are limited to the duration of the IFW. If this condition is satisfied, the system's performance becomes essentially noise-limited, rather than interference-limited. Ad hoc networks have been studied

(C) The Institution of Engineering and Technology 2007

doi:10.1049/iet-com:20050549

Paper first received 4th October 2005 and in revised form 10th January 2007

The authors are with School of Electronics and Computer Science, University of Southampton, Southampton SO17 1BJ, UK

E-mail: 1h@ecs.soton.ac.uk for decades and have evolved to sensor networks and mesh networks $[8,9]$, both of which potentially support a large number of nodes in the network. Although the application of LAS codes in cellular networks is limited by the low number of available spreading codes, this impediment is less restrictive in ad hoc networks, since the nodes only communicate with their neighbours. In this paper, we will focus our attention on a comparative study of LAS codes and traditional spreading sequences in direct sequence code division multiple access work is based on the following basic properties of ad hoc networks. First, there is no central base station (BS) control and hence no BS-aided power control and no synchronisation can be used. Secondly, each node has to have a low cost and hence global positioning system based synchronisation should ideally also be avoided. Finally, a cost-efficient implementation has to avoid complex multi-user detection or interference cancellation techniques, despite potentially encountering challenging near-far scenarios. As argued above, LAS codes have the potential to meet all the abovementioned requirements of ad hoc networks and the novel contribution of this paper is to quantify the benefits of LAS-CDMA ad hoc networks.

The outline of this paper is as follows. In Section 2 the system model of LAS DS-CDMA is described. The bit error rate (BER) performance of such a system is investigated in Section 3. Our comparative study of LAS codes and traditional spreading sequences is presented in Section 4. Finally, Section 5 provides our conclusions.

\section{System model}

For the sake of maintaining mathematical tractability and for gaining valuable insights into more realistic practical mobile wireless networks, we consider an infinite mesh of rectilinearly located nodes, as seen in Fig. 1. This idealised, but tractable topology allows us to examine the system's performance [10-12]. Its infinitely repeated structure results in a scenario having the highest possible interference 


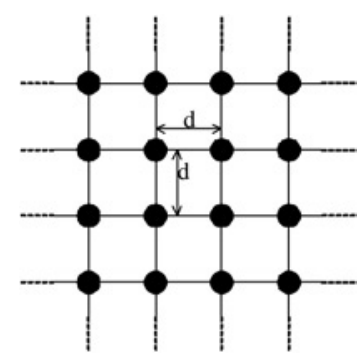

Fig. 1 Infinite mesh of rectilinear node topology, where the distance between any two adjacent nodes is $d$

and conveniently eliminates any system performance dependence on the size of the network. We use $(u d, v d)$ for denoting the location of nodes on the two-dimensional plane, where $u$ and $v$ are integers and $d$ is the minimum distance between any two adjacent nodes.

Every node in the network has four neighbours, connected by the edges, as seen in Fig. 1. We assume that the maximum affordable transmit power only supports communications between immediate neighbours. If a packet has to be sent from a source node to a destination node that is not an immediate neighbour, it has to be relayed by the intermediate nodes. Each node is assigned a spreading sequence for transmitting such that the shortest distance between any two nodes using the same spreading sequence is maximised. Each node is assumed to have an equal probability of transmitting or receiving signals to or from one of its neighbours. If a uniform geometric traffic generation model is used, the network is homogeneous and hence it is possible for us to study a single receiving reference node, rather than considering all nodes.

We will focus our attention on the system's single-hop performance under the above assumptions, rather than considering a particular routing strategy. We assume that each node transmits or receives with the probability of 0.5 , respectively, and that there is always sufficient data in the node' buffer. Without loss of generality, the receiving reference node is assumed to be located at the co-ordinate $(0,0)$ of the two-dimensional plane and receives from the transmitting node located at $(0, d)$. Hence all the other nodes are regarded as interferences, which interfere with a probability of 0.5 according to their transmission probability of 0.5 .

Consider $K$ simultaneously transmitting nodes in the context of wireless ad hoc networks, where each transmitting node transmits at the same constant power level $P_{\mathrm{t}}$, since cost-efficient ad hoc networks routinely dispense with power control. The multipath channel has the channel impulse response (CIR) of [13]

$$
h_{k}(t)=\sum_{l=0}^{L_{\mathrm{p}}-1} h_{k l} \delta\left(t-l T_{\mathrm{c}}\right) \mathrm{e}^{-j \vartheta_{k l}}
$$

where $h_{k}(t)$ is the complex-valued low-pass equivalent CIR of the multipath channel experienced by the transmitting node $k$. Furthermore, $L_{\mathrm{p}}$ is the total number of resolvable paths, which is assumed to be identical for all nodes, $h_{k l}$ is the fading envelope of the $l$ th path of the transmitting node $k, \delta(t)$ is the Dirac delta function, $T_{\mathrm{c}}$ is the chip duration and $\vartheta_{k l}$ is the phase-shift of the $l$ th path of the transmitting node $k$. In general, the phase angles $\left\{\boldsymbol{\vartheta}_{k l}\right\}$ are deemed to be independently and uniformly distributed in $[0,2 \pi)$, while the fading amplitudes $\left\{h_{k l}\right\}$ are independent Nakagami- $m$ distributed random variables having a probability distribution function of $p\left(h_{k l}\right)$ expressed as [13]

$$
p\left(h_{k l}\right)=\frac{2 m_{k l}^{m_{k l}} h_{k l}^{2 m_{k l}-1}}{\Gamma\left(m_{k l}\right) \Omega_{k l}^{m_{k l}}} \mathrm{e}^{-m_{k l} / \Omega_{k l}} h_{k l}^{2}
$$

In (2) $m_{k l}$ is the Nakagami- $m$ fading parameter, which characterises the severity of the fading on the $l$ th path of the transmitting node $k, \Omega_{k l}$ is the second moment of $h_{k l}$, which corresponds to the average path loss on the $l$ th path of the transmitting node $k[13]$, and $\Gamma(\cdot)$ is the gamma function [14]. We assume a negative exponentially decaying multipath intensity profile given by $[15,16]$

$$
\begin{aligned}
& \Omega_{k l}=\Omega_{k 0} \mathrm{e}^{-\eta l}, \quad k=1, \ldots, K \text { and } l=1, \ldots, L_{\mathrm{p}}-1 \\
& \Omega_{k 0}=E\left\{h_{k l}^{2}\right\}=r_{k}^{-\alpha}
\end{aligned}
$$

where $\eta>0$ is the rate of average power decay, which is assumed to be identical for all nodes, $r_{k}$ is the distance between the receiving reference node and the transmitting node $k$, whereas $\alpha>2$ is the path loss exponent [13], which is also assumed to be identical for all nodes.

The signal $x_{k}(t)$ transmitted by node $k$ is given by [16-19]

$$
x_{k}(t)=\sqrt{2 P_{t}} a_{k}(t) b_{k}(t) \mathrm{e}^{j \phi k}
$$

where $\phi_{k}$ is the carrier phase of the transmitting node $k$, which is assumed to be uniformly distributed in $[0,2 \pi)$. The spreading code $a_{k}(t)$ and the data signal $b_{k}(t)$ are defined as

$$
\begin{aligned}
& a_{k}(t)=\sum_{j=-\infty}^{\infty} a_{k j} \varphi_{T_{\mathrm{c}}}\left(t-j T_{\mathrm{c}}\right) \\
& b_{k}(t)=\sum_{j=-\infty}^{\infty} b_{k j} \varphi_{T_{\mathrm{s}}}\left(t-j T_{\mathrm{s}}\right)
\end{aligned}
$$

where $a_{k j} \in\{-1,1\}$ is a binary spreading sequence having a period $\mathrm{L}, b_{k j} \in\{-1,1\}$ is the binary data sequence, $\varphi_{T}(t)$ is the rectangular pulse having a period $T$ and $T_{\mathrm{s}}$ is the symbol duration satisfying $T_{\mathrm{s}}=L T_{\mathrm{c}}$.

The signal $y(t)$ received by the reference node is given node is given by

$$
\begin{aligned}
y(t)= & \sum_{k=1}^{K} \sum_{l=0}^{L_{\mathrm{p}}-1} \sqrt{2 P_{t}} a_{k}\left(t-l T_{\mathrm{c}}-\tau_{k}\right) \\
& \times b_{k}\left(t-l T_{\mathrm{c}}-\tau_{k}\right) h_{k l} \mathrm{e}^{j \theta_{k l}}+n(t)
\end{aligned}
$$

where $\tau_{k}$ is the propagation delay of the transmitting node $k$, $\theta_{k l}=\phi_{k}-\vartheta_{k l}$ is the phase-shift of the $l$ th path if the transmitting node $k$ at the receiving reference node and $n(t)$ is the complex-valued low-pass equivalent additive white Gaussian noise. Generally, $\theta_{k l}$ is independently and uniformly distributed in $[0,2 \pi)$ and $\tau_{k}$ is assumed to be uniformly distributed in the range of $\left[\tau_{k 0}, \tau_{k 0}+\tau_{\max }\right]$, where $\tau_{k 0}=\mathrm{r}_{k} / c$ is the shortest delay or line-of-sight (LOS) delay between the transmitting node $k$ and the reference node, where $c$ is the velocity of light. Furthermore, $\tau_{\max }$ is the maximum propagation delay relative to the LOS delay, which is assumed to be identical for all nodes.

\section{BER analysis}

Let us assume that the reference node is receiving signals from the transmitting node $q$ using maximal ratio combining (MRC) and the RAKE receiver combines a total of $L_{\mathrm{r}}$ branches. Then the output $Z_{q i}$ of its correlation receiver at 
the $i$ th branch is given by

$$
\begin{aligned}
Z_{q i} & =\operatorname{Re}\left\{\int_{i T_{\mathrm{c}}+\tau_{q}}^{T_{\mathrm{s}}+i T_{\mathrm{c}}+\tau_{q}} y(t) \alpha_{i} \mathrm{e}^{-j \theta_{q i}} a_{q}\left(t-i T_{\mathrm{c}}-\tau_{q}\right) \mathrm{d} t\right\} \\
& =D_{q i}+I_{(S) q i}+I_{(M) q i}+n_{q i}
\end{aligned}
$$

where $\alpha_{i}$ is the branch or finger weight of the RAKE receiver [16], $D_{q i}$ is the required signal, while $I_{(S) q i}$ is the MPI imposed by the other paths of node $q, I_{(M) q i}$ is the MAI incurred by other nodes and $n_{q i}$ conditioned on the finger weights $\left\{\alpha_{i}\right\}$ of the RAKE receiver and the $q$ th user's spreading sequence $a_{q}(t)$ [16].

The required signal $D_{q i}$ can be expressed as

$$
D_{q i}=\sqrt{2 P_{t}} T_{\mathrm{s}} b_{q 0} h_{q i} \alpha_{i}
$$

The MPI $I_{(S) q i}$ and its variance $\sigma_{(S) q i}^{2}$ are given by

$$
\begin{aligned}
I_{(S) q i}= & \sqrt{2 P_{t}} T_{\mathrm{c}} \alpha_{i} \sum_{\substack{l=0 \\
l \neq i}}^{L_{\mathrm{p}}-1} h_{q l} \cos \left(\theta_{q l}-\theta_{q i}\right) \\
& \times\left[b_{q,-1} c_{q q}(\xi-L)+b_{q 0} c_{q q}(\xi)\right] \\
\sigma_{(S) q i}^{2}= & P_{t} T_{\mathrm{c}}^{2} \alpha_{i}^{2} \sum_{\substack{l=0 \\
l \neq i}}^{L_{\mathrm{p}}-1} \Omega_{q 0} \mathrm{e}^{-\eta l}\left[c_{q q}^{2}(\xi-L)+c_{q q}^{2}(\xi)\right]
\end{aligned}
$$

where we have $\xi=l-i$, and $c_{k q}(\xi)$ is the discrete aperiodic cross-correlation function of the spreading sequences $\left\{a_{k j}\right.$ $\mid j=0, \ldots, L-1\}$ and $\left\{a_{q j} \mid j=0, \ldots, L-1\right\}$, or for short $a_{k j}$ and $a_{q j}$ which is defined as (5) in [18].

The MAI $I_{(M) q i}$ and its variance $\sigma_{(M) q i}^{2}$ are given by

$$
\begin{aligned}
I_{(M) q i}= & \sqrt{2 P} T_{\mathrm{c}} \alpha_{i} \sum_{\substack{k=1 \\
k \neq q}}^{K} \sum_{l=0}^{L_{\mathrm{p}}-1} h_{k l} \cos \left(\theta_{k l}-\theta_{q i}\right) \\
& \times\left[b_{k,-1} \rho_{k q}\left(\tau_{\mathrm{c}}\right)+b_{k 0} \varrho_{k q}\left(\tau_{\mathrm{c}}\right)\right] \\
\sigma_{(M) q i}^{2}= & P_{t} T_{\mathrm{c}}^{2} \alpha_{i}^{2} \sum_{\substack{k=1 \\
k \neq q}}^{K} \sum_{l=0}^{L_{\mathrm{p}}-1} \Omega_{k 0} \mathrm{e}^{-\eta l} \frac{T_{\mathrm{c}}^{2}}{\tau_{\max }^{2}} \\
& \times\left[\left.\sum_{j=\lambda_{-}}^{\lambda_{0}} S_{j}^{(1)}(t)\right|_{\max \left\{\tau_{-}-j, 0\right\}} ^{\min \left\{\tau_{0}-j, 1\right\}}\right. \\
& \left.-\left.\sum_{j=\lambda_{0}}^{\lambda_{+}} S_{j}^{(2)}(t)\right|_{\max \left\{\tau_{0}-j, 0\right\}} ^{\min \left\{\tau_{+}-j, 1\right\}}\right]
\end{aligned}
$$

where we have $\zeta=(l-i+j) \bmod L$ and the partial integral $\left.S_{j}^{(i)}(t)\right|_{t_{1}} ^{t_{2}}=S_{j}^{(i)}\left(t_{2}\right)-S_{j}^{(i)}\left(t_{1}\right)$, and $S_{j}^{(i)}(\mathrm{t})$ is given by

$$
\begin{aligned}
S_{j}^{(i)}(t)= & \frac{1}{4} w_{1}(\zeta) t^{4}+\frac{1}{3}\left[w_{1}(\zeta)\left(j-\tau_{i}\right)+2 w_{2}(\zeta)\right] t^{3} \\
& +\frac{1}{2}\left[2 w_{2}(\zeta)\left(j-\tau_{i}\right)+w_{3}(\zeta)\right] t^{2}+w_{3}(\zeta)\left(j-\tau_{i}\right) t \\
\tau_{i}= & \begin{cases}\tau_{-}, & \text {if } i=1 \\
\tau_{+}, & \text {if } i=2\end{cases}
\end{aligned}
$$

The coefficients $\omega_{1}(\zeta), \omega_{2}(\zeta)$ and $\omega_{3}(\zeta)$ in (15) are given by

$$
\begin{aligned}
w_{1}(\zeta)= & {\left[c_{k q}(\zeta+1-L)-c_{k q}(\zeta-L)\right]^{2} } \\
& +\left[c_{k q}(\zeta+1)-c_{k q}(\zeta)\right]^{2} \\
w_{2}(\zeta)= & c_{k q}(\zeta-L)\left[c_{k q}(\zeta+1-L)-c_{k q}(\zeta-L)\right] \\
& +c_{k q}(\zeta)\left[c_{k q}(\zeta+1)-c_{k q}(\zeta)\right] \\
w_{3}(\zeta)= & c_{k q}^{2}(\zeta-L)+c_{k q}^{2}(\zeta)
\end{aligned}
$$

The parameters $\tau_{-}, \tau_{0}, \tau_{+}$defining the integral area and their integer parts $\lambda_{-}, \lambda_{0}, \lambda_{+}$are given by

$$
\begin{array}{ll}
\tau_{-}=\frac{\tau_{k 0}-\tau_{q 0}-\tau_{\max }}{T_{\mathrm{c}}}, & \lambda_{-}=\left\lfloor\tau_{-}\right\rfloor \\
\tau_{0}=\frac{\tau_{k 0}-\tau_{q 0}}{T_{\mathrm{c}}}, & \lambda_{0}=\left\lfloor\lambda_{0}\right\rfloor \\
\tau_{+}=\frac{\tau_{k 0}-\tau_{q 0}-\tau_{\max }}{T_{\mathrm{c}}}, & \lambda_{+}=\left\lfloor\tau_{+}\right\rfloor
\end{array}
$$

The Gaussian noise $n_{q i}$ and its variance $\sigma_{(N) q i}^{2}$ are given by

$$
\begin{aligned}
n_{q i} & =\operatorname{Re}\left\{\alpha_{i} \int_{0}^{T_{\mathrm{s}}} n(t) a_{q}(t) \Psi(t) \mathrm{e}^{j \theta_{q i}} \mathrm{~d} t\right\} \\
\sigma_{(N) q i}^{2} & =\alpha_{i}^{2} N_{0} T_{\mathrm{s}}
\end{aligned}
$$

Consequently, the SINR encountered on the $i$ th path of transmitting node $q$ at the receiving reference node is denoted by $2 \gamma_{i}$ where $\gamma_{i}$ is given by

$$
\gamma_{i}=\frac{D_{q i}^{2}}{2\left[\sigma_{(S) q i}^{2}+\sigma_{(M) q i}^{2}+\sigma_{(N) q i}^{2}\right]}
$$

The term bit error probability (BEP) will be used interchangeably with the BER $P_{b \mid h}(\gamma)$, which is conditioned on the fading envelope vector $h$. When using binary phase shift keying (BPSK) for transmitting from node $n$ to the receiving reference node, the BEP is given by applying the Gaussian approximation as [20]

$$
P_{\mathrm{b}}(\boldsymbol{\gamma})=Q\left(\sqrt{\sum_{i=0}^{L_{\mathrm{r}}-1} 2 \gamma_{i}}\right)
$$

where $\gamma$ represents the vector constituted by $\left\{\gamma_{i}\right\}, L_{\mathrm{r}}$ is the total number of RAKE receiver branches and $Q(x)$ is the Gaussian $Q$-function. Fir the sake of conveniently evaluating the average BER, we will use an alternative definite integral of the form of $Q(x)$, which was formulated in (2) of [20].

Finally, the average BER at the reference node receiving from the transmitting node $q$ is given by [20]

$$
P_{\mathrm{b}}=\frac{1}{\pi} \int_{0}^{\pi / 2} \prod_{i=0}^{L_{\mathrm{r}}-1}\left(\frac{m_{i} \sin ^{2} \theta}{\bar{\gamma}_{i}+m_{i} \sin ^{2} \theta}\right)^{m_{i}} \mathrm{~d} \theta
$$

where $2 \bar{\gamma}_{i}$ is the average signal to interference-plus-noise ratio (SINR) of the transmitting node $q$ at the receiving reference node, and the SINR-related term $\bar{\gamma}_{i}$ in (22) is given by

$$
\bar{\gamma}_{i}=\left[\frac{Y_{S}(i)}{L^{2}}+\frac{Y_{M}(i)}{L^{2}}+\frac{1}{\gamma_{\mathrm{SNR}}}\right]^{-1} \mathrm{e}^{-\eta i}
$$

where $\Upsilon_{S}(i)$ and $Y_{M}(i)$ are the MPI and the MAI related terms, respectively, and $\gamma_{\mathrm{SNR}}$ is the received per-bit signal-to-noise ratio (SNR), which does not take into 
account the received interference power. They are formulated as

$$
\begin{aligned}
\Upsilon_{S}(i)= & \sum_{\substack{i=0 \\
l \neq i}}^{L_{\mathrm{p}}-1} \mathrm{e}^{-\eta l}\left[c_{q q}^{2}(\xi-L)+c_{q q}^{2}(\xi)\right] \\
\Upsilon_{(M)}(i)= & \sum_{\substack{k=1 \\
k \neq q}}^{K} \sum_{l=0}^{L_{\mathrm{p}}-1} \frac{\Omega_{k 0}}{\Omega_{q 0}} \mathrm{e}^{-\eta l} \frac{T_{\mathrm{c}}^{2}}{\tau_{\max }^{2}} \\
& \times\left[\left.\sum_{j=\lambda_{-}}^{\lambda_{0}} S_{j}^{(1)}(t)\right|_{\max \left\{\tau_{-}-j, 0\right\}} ^{\min \left\{\tau_{0}-j, 1\right\}}\right. \\
& \left.-\left.\sum_{j=\lambda_{0}}^{\lambda_{+}} S_{j}^{(2)}(t)\right|_{\max \left\{\tau_{0}-j, 0\right\}} ^{\min \left\{\tau_{+}-j, 1\right\}}\right] \\
\gamma_{\mathrm{SNR}}= & \frac{P_{\mathrm{t}} L T_{\mathrm{c}} \Omega_{q 0}}{N_{0}}
\end{aligned}
$$

\section{Performance of LAS DS-CDMA}

We will compare the achievable performance of a number of systems, namely that of quasi-synchronous LAS DS-CDMA and quasi-synchronous DS-CDMA using both Walsh-Hadamard (WH) codes and Orthogonal Gold (OG) codes, as well as asynchronous LAS DS-CDMA and asynchronous DS-CDMA using random random signature sequences. BPSK modulation is used in all of the above systems.

We investigate the attainable performance of the system using LAS-CDMA-2000-like physical layer parameters [21], where the corresponding spreading gain is $G=151$. For the sake of a fair comparison we whould need both WH and OG codes having a length of $L=151$ chips. However, no such codes exist, and the most similar ones are those having a length of $L=128$ and $L=256$ chips. When considering asynchronous DS-CDMA using random sequences, we assume that the spreading gain of the random sequences is the same as that of the LAS-CDMA-2000 codes, namely $G=151$, which is also equal to the length $L$ of the random signature sequences.

Fig. 2 shows that the BERs of all systems considered decrease, as the SNR increases. This can be readily seen from (23), where $P_{\mathrm{b}}(\gamma)$ is a monotonically decreasing function of $\gamma_{\mathrm{SNR}}$. However, it may also be concluded from (23) that increasing the transmitted power is only capable of mitigating the transmitted power substantially improves the achievable BER performance of the quasi-synchronous LAS DS-CDMA system. The quasi-synchronous DS-CDMA systems using both 256-chip and 128-chip WH codes exhibit a poor performance owing to their high auto-correlation function side-lobes. In contrast, the quasisynchronous DS-CDMA systems using 256-chip and 128-chip OG codes exhibit a better performance, as a benefit of their good correlation properties. It is worth noting that the performance of quasi-synchronous DS-CADA systems is dominated by a limited number of high cross-correlation values found between the spreading sequences used by the desired node and the interferers at a time-displacement of $\xi=0$. Unfortunately, there are several OG codes having a high cross-correlation value at or near the displacement of $\xi=0$. These cross-correlation values are proportional to the spreading gain of the code concerned. This is the reason that the quasi-synchronous DS-CDMA systems using 256-chip and 128-chip OG

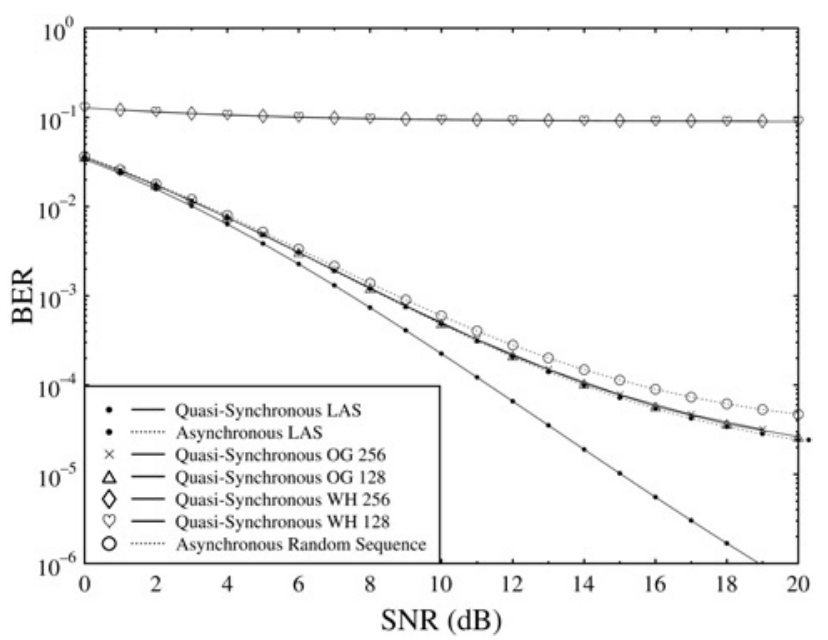

Fig. 2 BER against the received per-bit $S N R \gamma_{S N R}$

All nodes are assumed to transmit with a probability of 0.5 at the same power level $P_{t}$

An infinite number of nodes are placed on a simple infinite rectilinear grid, with the minimum distance between adjacent nodes $d=30 \mathrm{~m}$.

Maximum propagation delay is $\tau_{\max }=2 T_{\mathrm{c}}$ and the chip duration is $2 \mu \mathrm{s}$

Total number of resolvable paths is $L_{\mathrm{p}}=4$, and the RAKE receiver combines $L_{\mathrm{r}}=3$ paths

IFW width is $\iota=3$

Rate of average negative exponential power decay is $\eta=0.2$

Nakagami- $m$ fading parameter is $m=1.0$

Pathloss exponent is $\alpha=4.0$

These results were evaluated from (22)

exhibit a similar performance. The asynchronous LAS DS-CDMA system performs slightly better than the asynchronous DS-CDMA system using random sequences, as a consequence of its lower MPI resulting from its IFW, given the parameters of $L_{\mathrm{p}}=4$ and $\iota=3$. The quasisynchronous LAS DS-CDMA system performs best, since its IFW cancels most of the MPI, given the parameters of $L_{\mathrm{p}}=4$ and $\iota=3$. Hence the quasi-synchronous LAS DS-CDMA system becomes essentially noise-limited, rather than interference-limited, which is also the reason that it benefits most from increasing the transmitted power.

In order to demonstrate the capability of LAS codes in terms of mitigating both MPI and MAI, we let $\gamma_{\mathrm{SNR}} \rightarrow \infty$ in Fig. 3. Fig. 3 shows that the BER of the quasisynchronous LAS DS-CDMA system degrades, as the maximum propagation delay increases, since the effects of less accurate synchronization inflict a higher MAI. Even the asynchronous LAS DS-CDMA system performs slightly better than the asynchronous DS-CDMA arrangement using random sequences, since most of the MPI is eliminated by the asynchronous LAS DS-CDMA scheme, given the parameters of $L_{\mathrm{p}}=4$ and $\iota=3$. The maximum propagation delay has a marginal effect on the quasi-synchronous DS-CDMA scheme using WH codes and the system having a higher spreading gain has almost no advantages, since the MPI dominates the achievable performance, as it can be concluded from their associated auto-correlation and cross-correlation functions. The quasi-synchronous system using 256-chip OG codes performs best at a large maximum propagation delay, mainly as a consequence of its higher spreading gain, while the LAS codes exhibit increased auto-correlation side-lobes outside the IFW.

In our further investigations, the system's performance for various $m$ values exhibits similar performance trends to the $m=1$ scenario, since the performance is mainly dependent on the correlation properties of the spreading 


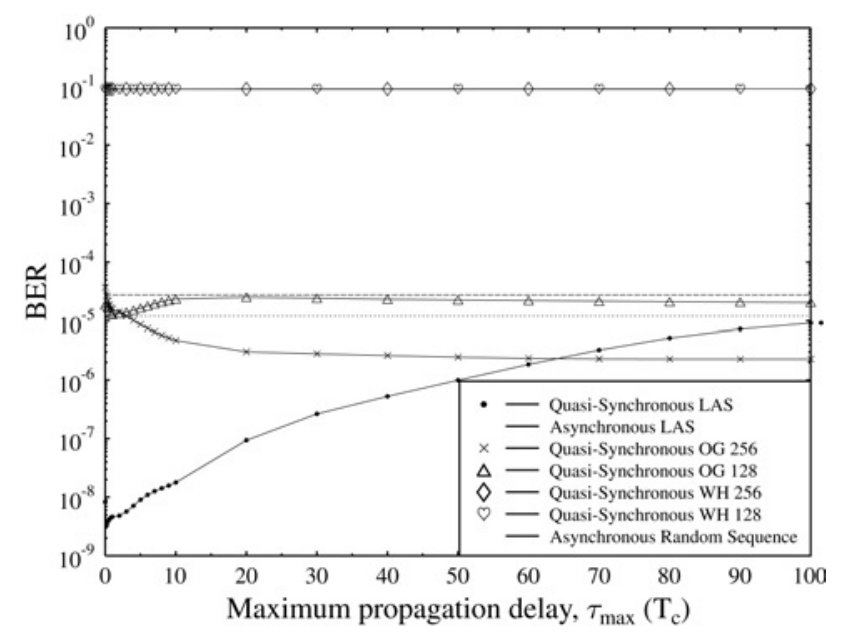

Fig. 3 BER against the maximum propagation delay $\tau_{\max }$ expressed in terms of the chip duration $T_{c}$

All nodes are assumed to transmit with a probability of 0.5 at the same power level $P_{t}$

An infinite number of nodes are placed on a simple infinite rectilinear grid, with the minimum distance between adjacent nodes $d=30 \mathrm{~m}$

Chip duration is $2 \mu \mathrm{s}$

Noise is ignored, that is $\gamma_{\mathrm{SNR}} \rightarrow \infty$

Total number of resolvable paths is $L_{\mathrm{p}}=4$, and the RAKE receiver combines $L_{\mathrm{r}}=3$ paths

IFW width is $\iota=3$

Rate of average negative exponential power decay is $\eta=0.2$

Nakagami- $m$ fading parameter is $m=1.0$

Pathloss exponent is $\alpha=4.0$

These results were evaluated from (22)

sequences and less critically on the Nakagami fading parameter, as suggested by our analysis in Section 3. However, when the number of resolvable multipath components, $L_{\mathrm{p}}$, is high, LAS DS-CDMA inevitably encounters serious MPI and MAI. There is a large BET discrepancy between $L_{\mathrm{p}}=8$ and $L_{\mathrm{p}}=12$, because there are high auto-correlation side-lobes at $\xi= \pm 8$ outside the IFW. On the other hand, increasing the total number of RAKE receiver branches dramatically improves the performance of the quasi-synchronous LAS DS-CDMA system, when the total number of resolvable paths is assumed to be $L_{\mathrm{p}}=4$. This performance improvement is attributed to the RAKE receiver, which benefits from the associated time-diversity.

\section{Conclusion}

In conclusion, LAS DS-CDMA was investigated in the context of an ad hoc network obeying an infinite rectilinear mesh topology. The system exhibits a significantly better performance than the family of traditional spreading sequences used in a quasi-synchronous DS-CDMA scenario having a low number of resolvable multipath components and a sufficiently high number of RAKE receiver branches.

\section{References}

1 Li, D.: 'The perspectives of large area synchronous CDMA technology for the fourth-generation mobile radio', IEEE Commun. Mag., 2003, 41, (3), pp. 114-118

2 Lee, W.C.Y.: 'Analysis and realization of a physical CDD system', Wiley J. Wirel. Commun. Mobile Comput., 2003, 3, (5), pp. 571-583

$3 \mathrm{Li}$, D.: 'A high spectrum efficient multiple access code'. in Fifth Asia-Pacific Conf. on Communications and Fourth Optoelectonics and Communication Conf., Beijing, China, October 1999), vol. 1, pp. 598-605

4 Wei, H., and Hanzo, L.: 'On the uplink performance of LAS-CDMA', IEEE Trans. Wirel. Commun., 2006, 5, (5), pp. 1187-1196

5 Stanczak, S., Boche, H., and Haardt, M.: 'Are LAS-codes a miracle ?'. IEEE Global Telecommunications Conf., San Antonio, Texas, USA, vol. 1, November 2001, pp. 589-593

6 Choi, B.-J., and Hanzo, L.: 'On the design of LAS spreading codes'. in IEEE 56th Vehicular Technology Conference 2002-Fall, Vancouver, British Columbia, Canada, September 2002, pp. 2172-2176

7 Ni, S., Wei, H., Blogh, J.S., and Hanzo, L.: 'Network performance of asynchronous UTRA-like FDD/CDMA systems using loosely synchronised spreading codes'. IEEE 58th Vehicular Technology Conf. 2003-Fall, Orlando, Florida, USA, October 2003, vol. 2, pp. 1359-1363

8 Niculescu, D.: 'Communication paradigms for sensor network', IEEE Commun. Mag., 2005, 43, (3), pp. 116-122

9 Bruno, R., Conti, M., and Gregori, E.: 'Mesh networks: commodity multihop ad hoc networks', IEEE Commun. Mag., 2005, 43, (3), pp. $123-131$

10 Silvester, J.A., and Kleinrock, L.: 'On the capacity of multihop slotted networks with regular structure', IEEE Trans. Commun., 1983, 31, (8), pp. 974-982

11 Xie, L.-L., and Kumar, P.: 'A network information theory for wireless connuniction: scaling laws and optimal operation', IEEE Trans. Inf. Theory, 2004, 50, (5), pp. 748-767

12 Hekmat, R., and Mieghem, P.V.: 'Interference in wireless multi-hop ad-hoc networks and its effect on network capacity', Wirel. Netw., 2004, 10, (4), pp. 389-399

13 Proakis, J.G.: 'Digital communication' (McGraw-Hill Companies, Inc, 2001, 4th edn.)

14 Abramowitz, M., and Stegun, I.A.: 'Handbook of mathematical functions, with formulas, graphs, and mathematical tables' (Department of Commerce, USA, 1972)

15 Turin, G.L., Clapp, F.D., Johnston, T.L., Fine, S.B., and Lavry, D.: ‘A statistical model of urban multipath propagation', IEEE Tans. Veh. Technol., 1972, 21, (1), pp. 1-9

16 Eng, T., and Milstein, L.B.: 'Coherent DS-CDMA performance in Nakagami multipath fading', IEEE Trans. Commun., 1995, 43, (2/ 3/4), pp. 1134-1143

17 Geraniotis, E., and Ghaffari, B.: 'Performance of binary and quaternary direct-sequence spread-spectrum maultiple-access systems with random signature sequence', IEEE Trans. Commun., 1991, 39, (5), pp. 713-724

18 Lehnert, J.S., and Pursley, M.B.: 'Error probabilities for binary direct-sequence spread-spectrum communications with random signature sequences', IEEE Trans. Commun., 1987, 35, (1), pp. 87-98

19 Pursley, M.B.: 'Performance evaluation for phase-coded spread-spectrum multiple-access communication-part I: system analysis', IEEE Trans. Commun., 1977, 25, (8), pp. 795-799

20 Simon, M.K., and Alouini, M.-S.: 'A unified approach to the performance analysis of digital communication over generalized fading channel', IEEE Proc., 1998, 86, (9), pp. 1860-1877

21 'Physical layer specification for LAS-2000', June 2000, CWTS/China 